\title{
EchoGéo
}

45 | 2018

Déclinaisons géographiques du changement social en Iran

\section{Dynamiques migratoires des espaces ruraux en Iran : une étude de cas}

\section{Amir Amiri}

\section{(2) OpenEdition}

\section{Journals}

Édition électronique

URL : https://journals.openedition.org/echogeo/16006

DOI : 10.4000/echogeo.16006

ISSN : 1963-1197

Éditeur

Pôle de recherche pour l'organisation et la diffusion de l'information géographique (CNRS UMR 8586)

Référence électronique

Amir Amiri, «Dynamiques migratoires des espaces ruraux en Iran : une étude de cas », EchoGéo [En ligne], 45 | 2018, mis en ligne le 05 novembre 2018, consulté le 10 août 2021. URL : http:// journals.openedition.org/echogeo/16006; DOI : https://doi.org/10.4000/echogeo.16006

Ce document a été généré automatiquement le 10 août 2021.

EchoGéo est mis à disposition selon les termes de la licence Creative Commons Attribution - Pas d'Utilisation Commerciale - Pas de Modification 4.0 International (CC BY-NC-ND) 


\title{
Dynamiques migratoires des espaces ruraux en Iran : une étude de cas
}

\author{
Amir Amiri
}

\section{Introduction}

1 La redistribution de la population du fait des mouvements migratoires est restée une caractéristique importante et reconnue des dynamiques démographiques en Iran, tout comme dans d'autres pays en développement. D'un côté, sur le plan de la migration interne, en moyenne un million de personnes ont migré chaque année à l'intérieur du pays au cours des trois dernières décennies. Cette tendance témoigne de l'émergence de la migration interne en tant que problème sociodémographique significatif, qui a des conséquences sur de multiples aspects démographiques, sociaux, économiques et politiques du pays (Mahmoudian et Ghassemi, 2014). La migration interne a eu lieu principalement depuis les régions les moins développées vers des zones plus attractives. Cela a conduit à un déséquilibre démographique. Au cours de notre enquête de terrain dans le Nord-Ouest du pays, il est apparu que certaines communes rurales étaient en train de se dépeupler, voyant leur rôle traditionnel dans la production agroalimentaire en cours de disparition et exprimant donc le besoin d'un apport démographique jeune. De l'autre côté, sur le plan migratoire, nous avons rencontré des Afghans installés dans ces zones rurales, alors qu'il est plus fréquent d'observer la présence d'Afghans dans les grandes villes iraniennes (voir illustrations 1 et 2). Nous proposons donc d'interroger, dans le contexte étudié, le lien entre ces deux catégories migratoires, à savoir la migration interne et internationale.

2 Pour cela, cette étude s'appuie en premier lieu sur des entretiens effectués auprès de populations locales et de travailleurs afghans dans des zones rurales ainsi que dans deux grandes villes de destination pour les migrants : Téhéran et Karaj. Elle s'appuie en second lieu sur les données quantitatives issues du recensement iranien. L'analyse portera d'abord sur les principales raisons invoquées par les enquêtés pour motiver leur migration et décrire leur activité professionnelle, puis nous examinerons le rôle de 
la migration rurale iranienne dans le dépeuplement des villages et l'urbanisation galopante, ainsi que son rôle comme possible facteur explicatif de la mobilité afghane.

Dans la mesure où cet article tente de croiser deux catégories de la migration, il est nécessaire de contextualiser et de donner un aperçu de la migration interne iranienne et de la migration afghane en Iran.

Illustration 1 - Part de la population afghane par rapport à la population iranienne dans les milieux ruraux, d'après les résultats du recensement de 2016

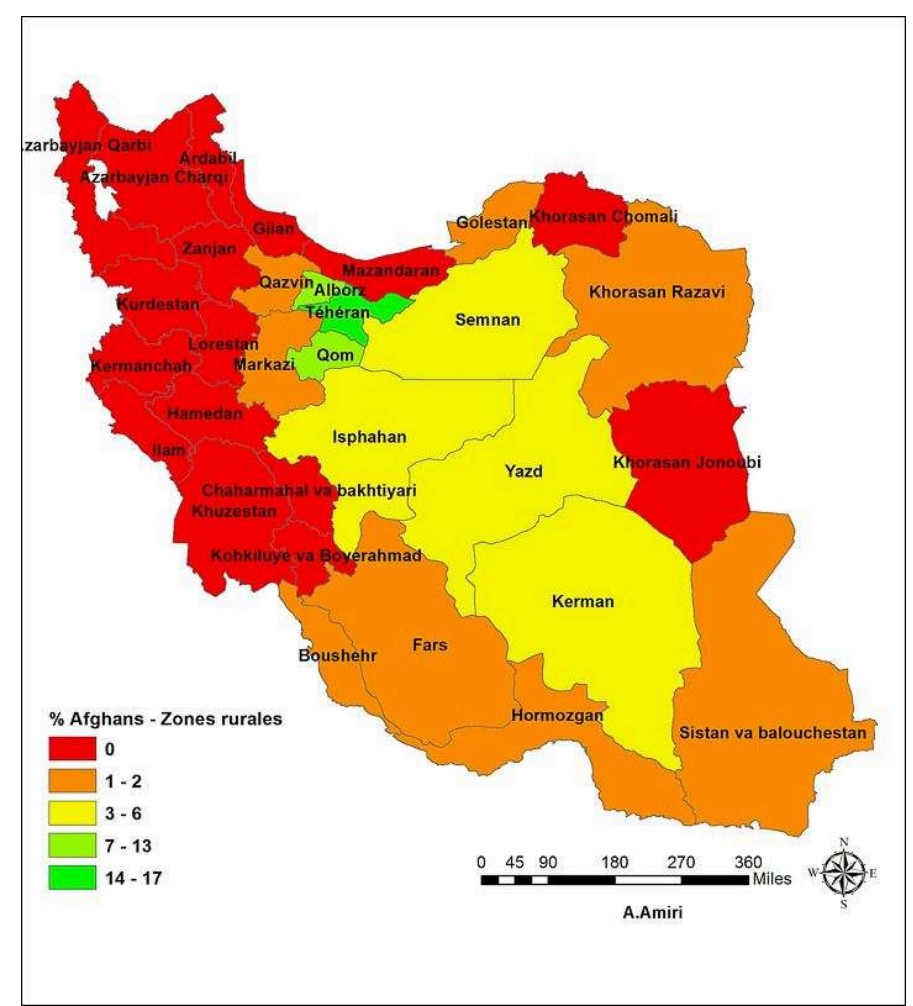

Source : carte réalisée par l'auteur à partir des données du recensement général de la population et de l'habitat, 2016. 
Illustration 2 - Part de la population afghane par rapport à la population iranienne dans les milieux urbains, d'après les résultats du recensement de 2016

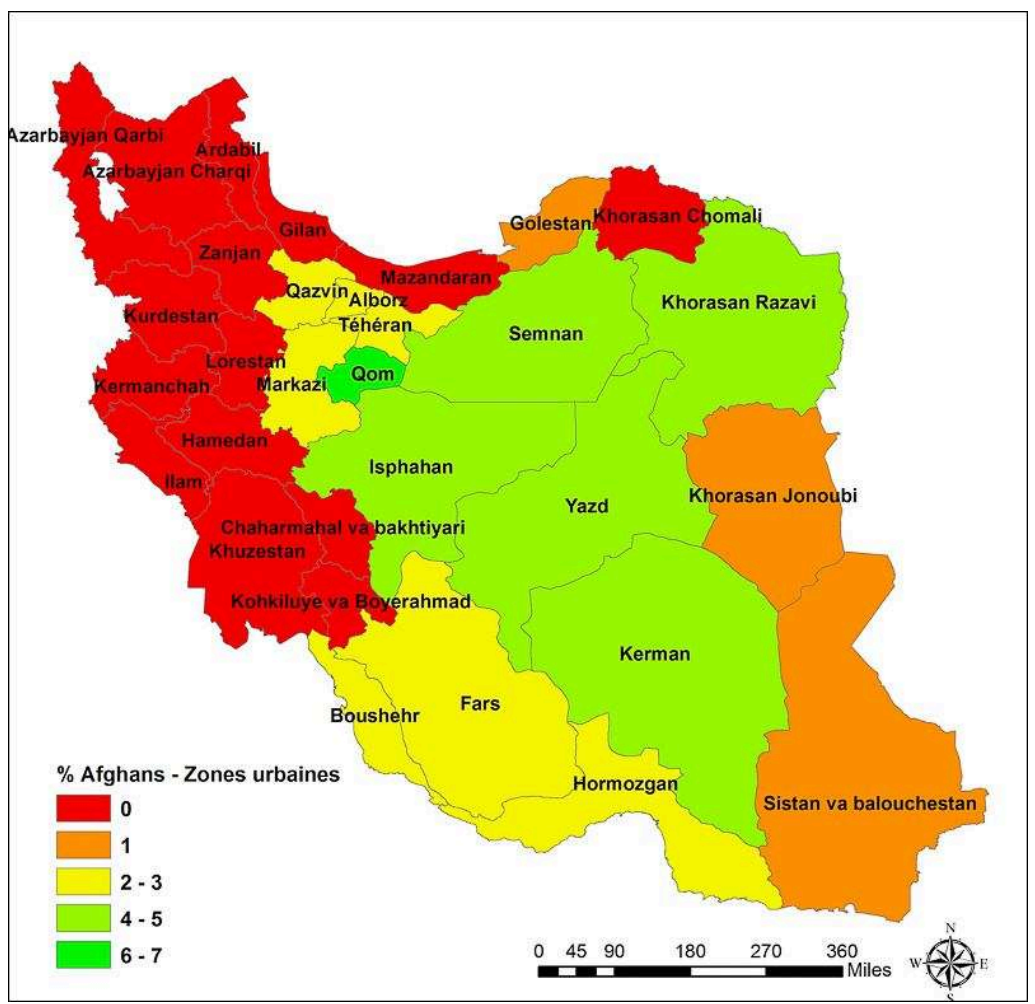

Source : carte réalisée par l'auteur à partir des données du recensement général de la population et de l'habitat, 2016.

\section{Contextualisation de l'étude}

4 Cette section vise à contextualiser la recherche en replaçant, en premier lieu, les migrations rurales-urbaines en Iran et, en deuxième lieu, l'immigration afghane vers le pays dans leur perspective historique.

\section{La migration interne et l'urbanisation}

5 Les mobilités résidentielles de la population ont profondément marqué l'Iran par leur ampleur territoriale. Ce mouvement de migrations internes a pu s'opérer du milieu rural vers le milieu urbain ou inversement ou bien encore intervenir de manière interurbaine ou entre communes rurales. Toutefois, il convient de noter que les mouvements de population les plus fréquemment observés se font des villages vers les villes ou de petites villes vers les grandes. L'Iran, à l'instar de nombreux pays en développement, a connu une forte progression de sa population urbaine, sous l'effet de l'accroissement de la mobilité interne.

6 Cela s'explique par de profondes transformations du marché du travail, ceci dès la "Révolution Blanche»1, initiée en 1963. Appliquée d'en haut, avant la révolution, elle s'est en particulier accompagnée de "réformes agraires» aussi bien que du développement déséquilibré des zones rurales et des zones urbaines, entraînant un dépeuplement villageois affectant directement le secteur agricole, confronté à une 
pénurie de main-d'œuvre locale. Certes, après la Révolution islamique, l'une des politiques populaires gouvernementales visait à inverser l'exode rural en encourageant les migrants à retourner au village, en soutenant les organisations coopératives rurales indépendantes et en mettant l'accent sur la revitalisation rurale et l'indépendance agricole dans un processus de débureaucratisation ${ }^{2}$, et de redistribution des terres parmi les paysans sans terre. Mais cette tendance politique semble avoir changé vers la fin de 1982. Alors que l'indépendance des organisations paysannes a été réduite par la bureaucratisation, leurs griefs sont renvoyés à la bureaucratie judiciaire, et le soutien gouvernemental a aussi progressivement changé en faveur de la grande propriété (Farazmand, 2001). Le résultat a donc été la reprise de la migration vers les villes.

D'après les résultats du dernier recensement général de la population et de l'habitat, réalisé en 2016, le taux d'urbanisation est passé de $69 \%$ en 2006 à $71 \%$ en 2011 puis $74 \%$ en 2016. Le taux d'urbanisation de la population de l'Iran s'est ainsi accru d'une manière considérable d'un recensement à l'autre. Si, en 1956, un peu moins d'un tiers de la population, soit $31,67 \%$, vivait dans les zones urbaines, au cours des deux dernières décennies, les proportions de population urbaine et rurale se sont inversées (illustrations 3 et 4). En 2006, plus des deux tiers (68,56\%) de la population vivaient dans les zones urbaines.

L'impact de cette tendance est illustré par différents exemples de pyramides des âges des populations urbaine et rurale (voir infra, illustrations 10 à 13). Une simple comparaison des pyramides de ces deux catégories montre de quelle façon la population rurale a diminué.

Illustration 3 - Taux de croissance annuelle (en \%) de la population, 1956-2011

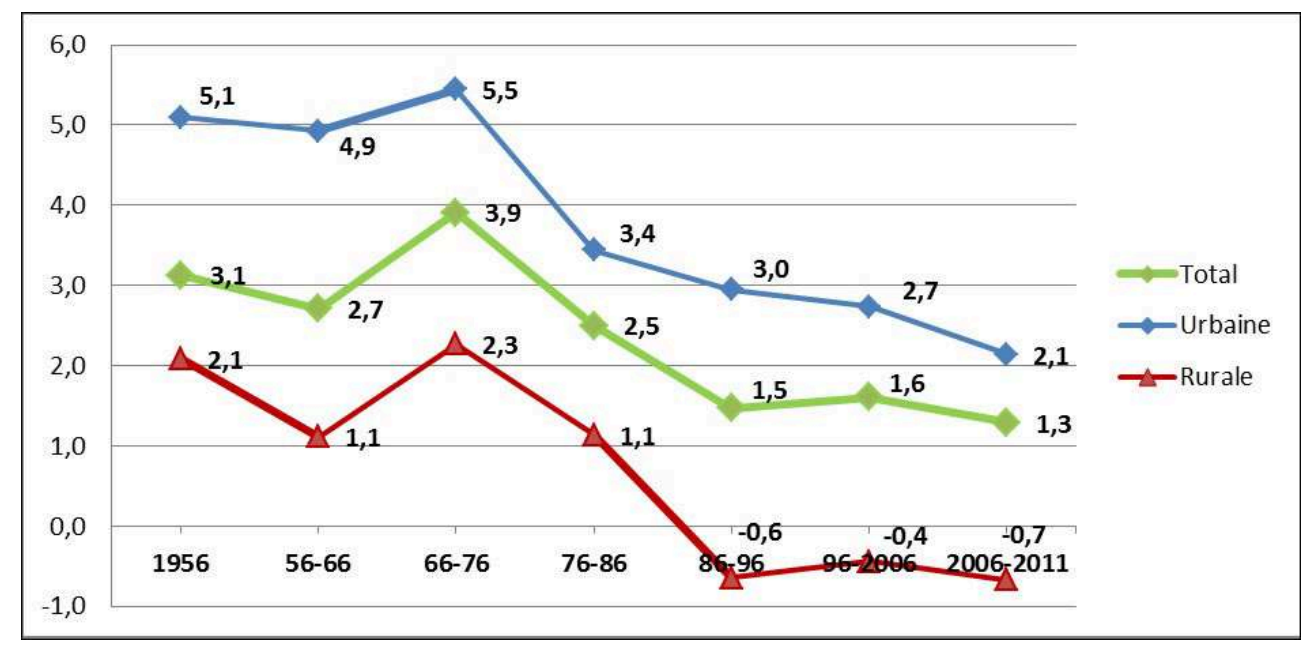

Sources : Centre de statistiques d'Iran, recensements de 1956 à 2011. 
Illustration 4 - Proportion de la population urbaine et rurale en Iran de 1956 à 2011 (en \%)

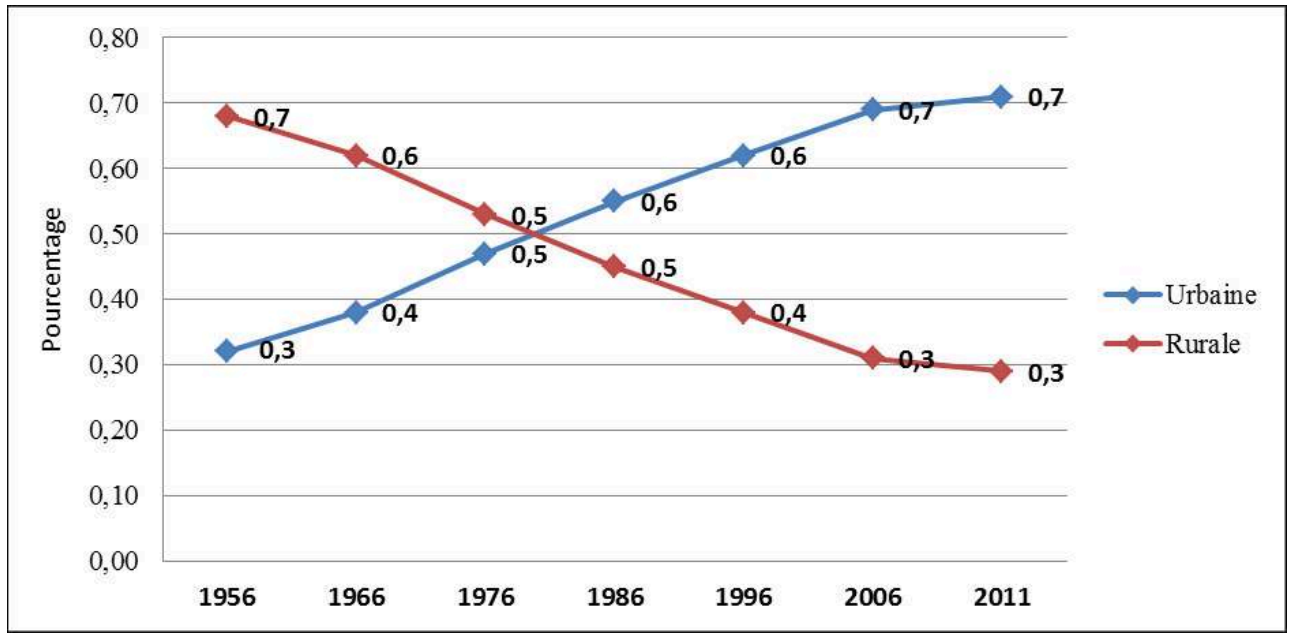

Sources : Centre de statistiques d'Iran, recensements de 1956 à 2011.

9 Les résultats des recensements permettent de connaître les effectifs de migrants, c'està-dire d'individus qui résidaient dans une autre commune, un autre département ou une autre région à la date du précédent recensement, 5 ou 10 ans auparavant. On observe ainsi qu'en termes de solde migratoire ${ }^{3}$, au cours des années 1996-2006, la région de Téhéran a été la plus attractive et bénéficiait de soldes migratoires internes très positifs; en effet cette région est bien équipée en infrastructures et dispose d'un marché du travail étendu et varié. De même, les régions de Khorasan Razavi, Mazandran, Gilan, Isfahan, Yazd, Boushehr et Qom bénéficiaient de soldes migratoires positifs ainsi que de taux annuels de migration nette positifs (illustration 5), alors que d'autres régions étaient globalement déficitaires, avec un solde migratoire négatif. $\mathrm{Au}$ cours des années 2006-2011 (illustration 6), on observe certaines modifications. Par exemple, le solde de la région de Téhéran devient négatif (pour la première fois). C'est le cas aussi, pour la région de Khorasan Razavi notamment. 
Illustration 5 - Taux annuels de migration nette (pour 1000 habitants), 1996-2006

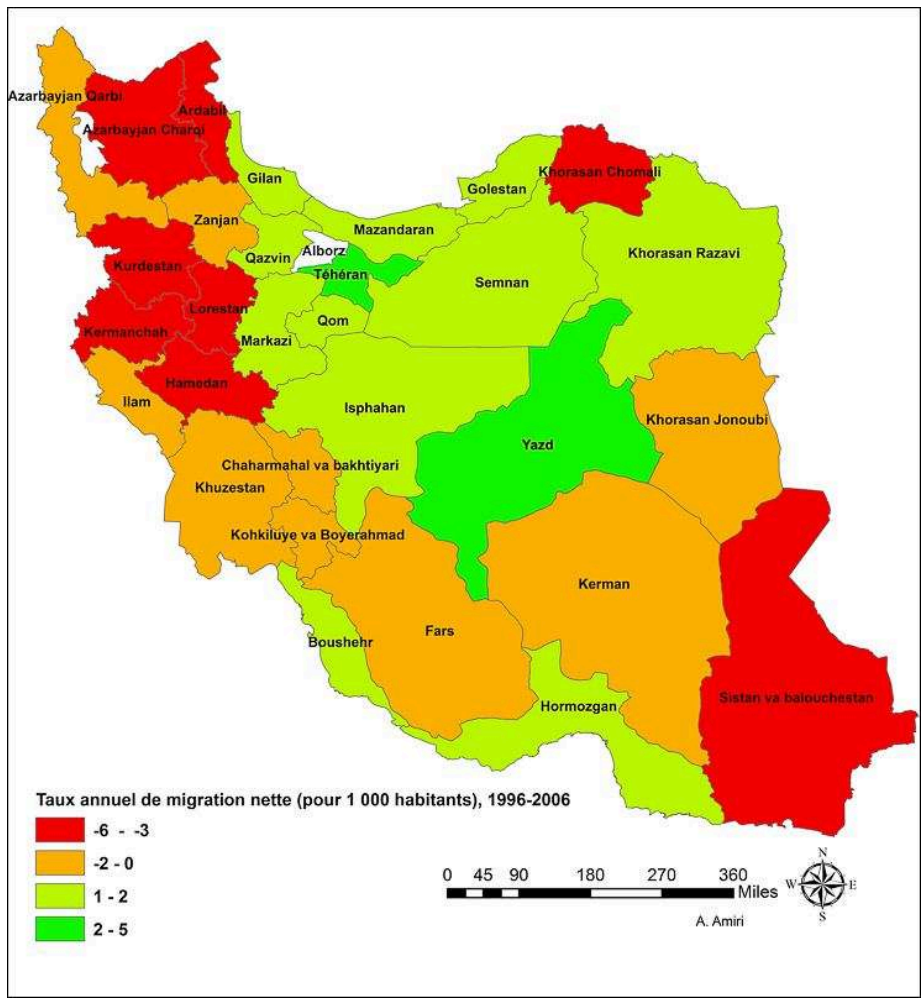

Source : carte réalisée par l'auteur à partir des données du recensement général de la population et de l'habitat, 1996- 2006. 


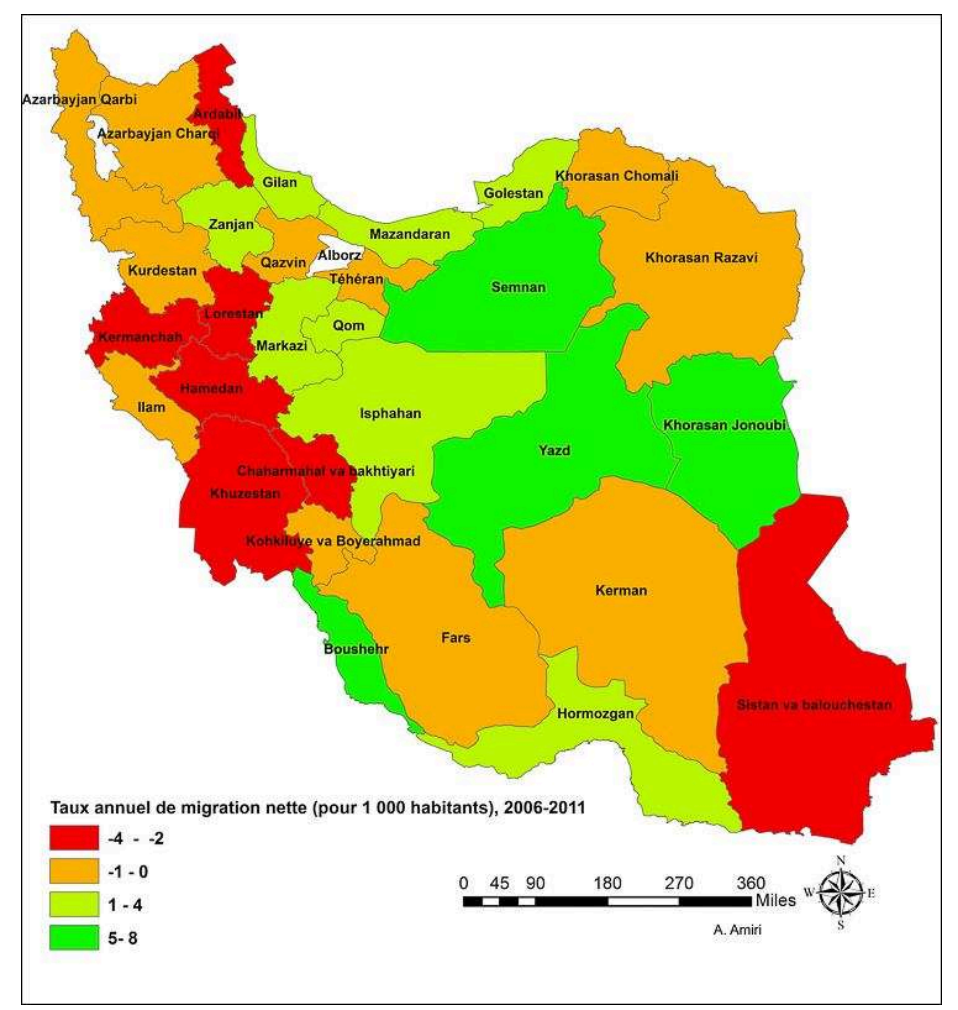

Source : carte réalisée par l'auteur à partir des données du recensement général de la population et de l'habitat, 2006-2011.

Il importe de noter que cette augmentation rapide du nombre de citadins d'un pays en développement a des implications importantes aussi bien en termes d'opportunités que de défis. L'étude des conditions de migration et d'urbanisation en Iran est révélatrice des disparités économiques et sociales des régions. L'intensité et le flux incessant des migrations rurales-urbaines ont également rendu la majorité des zones rurales sujettes à une dévitalisation durable (Mahmoudian, 2016). La migration rurale a également intensifié le sous-développement en privant les zones rurales de la population jeune ; la plupart des non-migrants (principalement des femmes et des personnes âgées) résidant dans les zones rurales manquent d'équipements économiques et sociaux adéquats (Mahmoudian et Ghasemi-Ardehaee, 2014). L'étalement urbain déchainé et l'urbanisation incontrôlée, en particulier dans les très grandes villes, ont eu des répercussions défavorables en termes de dégradation de l'environnement, et les migrants (pour la plupart des habitants des périphéries urbaines) souffrent d'un manque d'équipements de bien-être appropriés dans la ville de destination (Ibid.).

\section{La migration des Afghans}

11 Depuis quatre décennies, parmi les flux migratoires régionaux, les exodes massifs et de longue durée prédominent et, parmi eux, le déplacement des Afghans figure toujours au premier rang ${ }^{4}$. Les principales vagues de départ des populations afghanes sont le reflet d'un contexte de crises aiguës, de la violence politique, mais aussi des conditions géographiques et climatiques difficiles dans ce pays (notamment la sécheresse ou les contraintes des régions montagneuses rudes). Parmi ces crises, se sont succédés le coup 
d'État communiste en 1978, l'intervention soviétique en 1979, la chute du régime prosoviétique puis les combats entre les multiples factions de la résistance à partir de 1992, enfin l'arrivée des talibans au pouvoir en 1996, puis l'intervention des États-Unis et l'installation du nouveau gouvernement (2001). Du fait de ces crises multiples et d'autres facteurs sociodémographiques, l'Afghanistan a été pendant plus de trois décennies la plus grande source de demandeurs d'asile du monde. Le rapport du HCR en 2013 montre que, en moyenne, un réfugié sur quatre dans le monde est Afghan, $95 \%$ de ces réfugiés vivent au Pakistan ou en Iran. Ces proportions invitent à s'interroger sur les particularités de cet exode et sur ses répercussions démographiques, économiques et sociales, tant dans les lieux de départ que dans les espaces d'accueil ${ }^{5}$.

D'une part, les conflits internes, et d'autre part, les interventions étrangères dans l'Afghanistan contemporain ont fonctionné comme un générateur de déplacements renouvelés, internes et externes, de la population. Dans le même temps, la migration des Afghans recouvre également d'autres catégories, notamment la migration circulaire et saisonnière lorsque la destination est un pays voisin, notamment l'Iran et le Pakistan. Ainsi, historiquement, la migration a fait partie intégrante de la vie et des stratégies de survie des Afghans en lien avec des catastrophes naturelles comme les sécheresses et leurs effets sur les activités saisonnières de cueillette et de récolte.

Sur cette base, chronologiquement, l'histoire de l'immigration afghane vers l'Iran peut être divisée en quatre phases. La première phase de mouvements court du XIXe siècle jusqu'aux années 1970. La deuxième et la troisième phases sont caractérisées par un afflux important de populations issues de déplacements forcés au cours de la période de 1978 à 2001, inscrivant l'Iran au dixième rang mondial des pays d'accueil de réfugiés. En fait, dans cette période, la première vague importante de migration forcée de population afghane a été déclenchée et s'est amplifiée suite au conflit qu'a connu l'Afghanistan après le coup d'État communiste de 1978 et l'intervention soviétique de 1979 jusqu'en 1992. Les déplacements de population ont continué à se produire après la chute des communistes en avril 1992 par les partis de la résistance (Moudjahidins) et lorsqu'elles s'opposaient rapidement les unes aux autres. Puis la montée des talibans entre 1994-2001 a provoqué une autre grande vague de mouvement de population. Une quatrième phase a eu lieu à partir de 2001, en raison de l'intervention des États-Unis et de l'installation du nouveau gouvernement. Pour cette dernière phase, il faut souligner que les flux mixtes (comprenant des migrants et des demandeurs d'asile), sont plus importants 6 .

14 Au cours de ces différentes phases, le flux de réfugiés et d'immigrés afghans en Iran a varié, mais la proportion de population afghane en Iran, elle, persiste et ne varie pas de manière significative (illustration 7). En effet, les flux continuent dans deux sens, à savoir: le rapatriement (volontaire ou assisté) vers l'Afghanistan et les vagues des nouveaux arrivants vers l'Iran se conjuguent. Année après année, de nouveaux chiffres viennent en témoigner (illustration 8). 
Illustration 7 - Évolution de la population étrangère en Iran depuis 1986
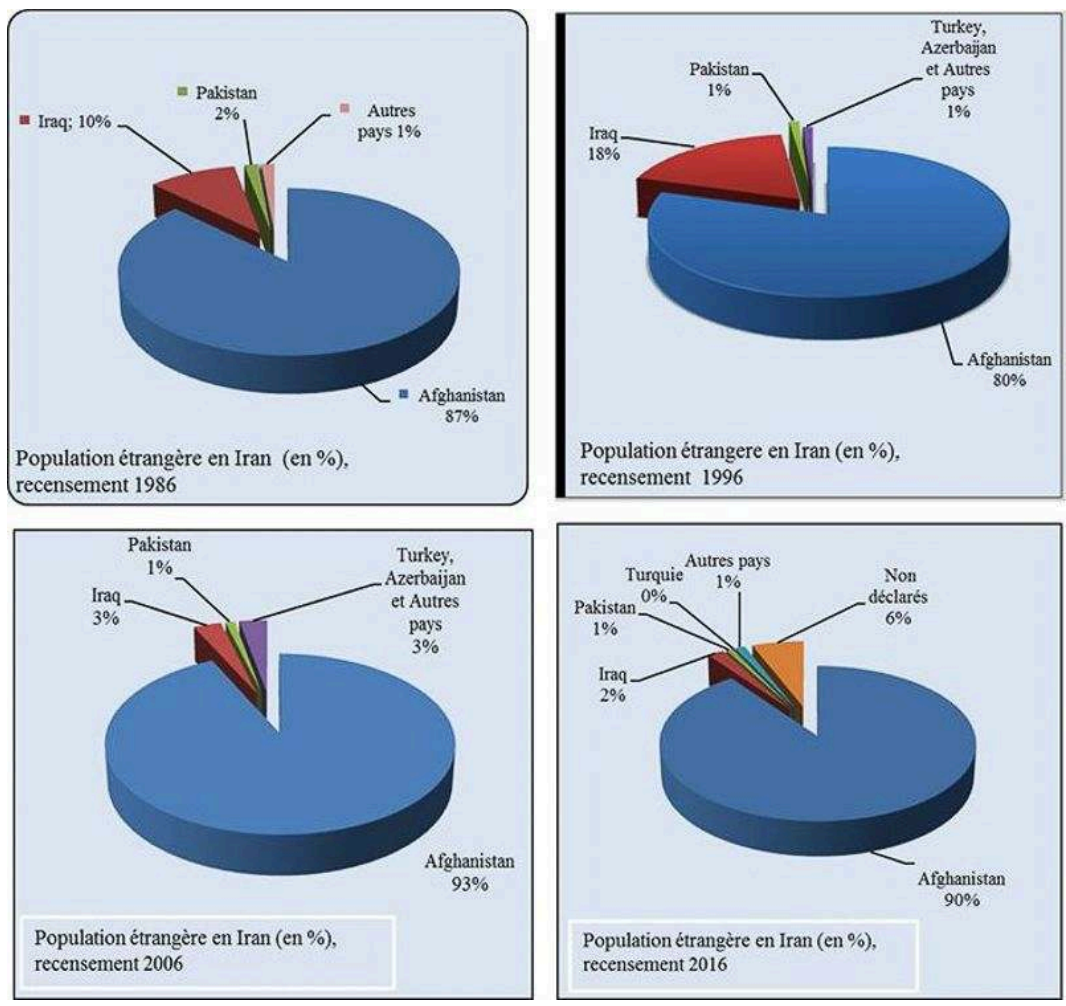

Sources : Centre de statistiques d'Iran, recensements décennaux de 1986 à 2016.

Illustration 8 - Effectif de réfugiés afghans en Iran, 1980-2008

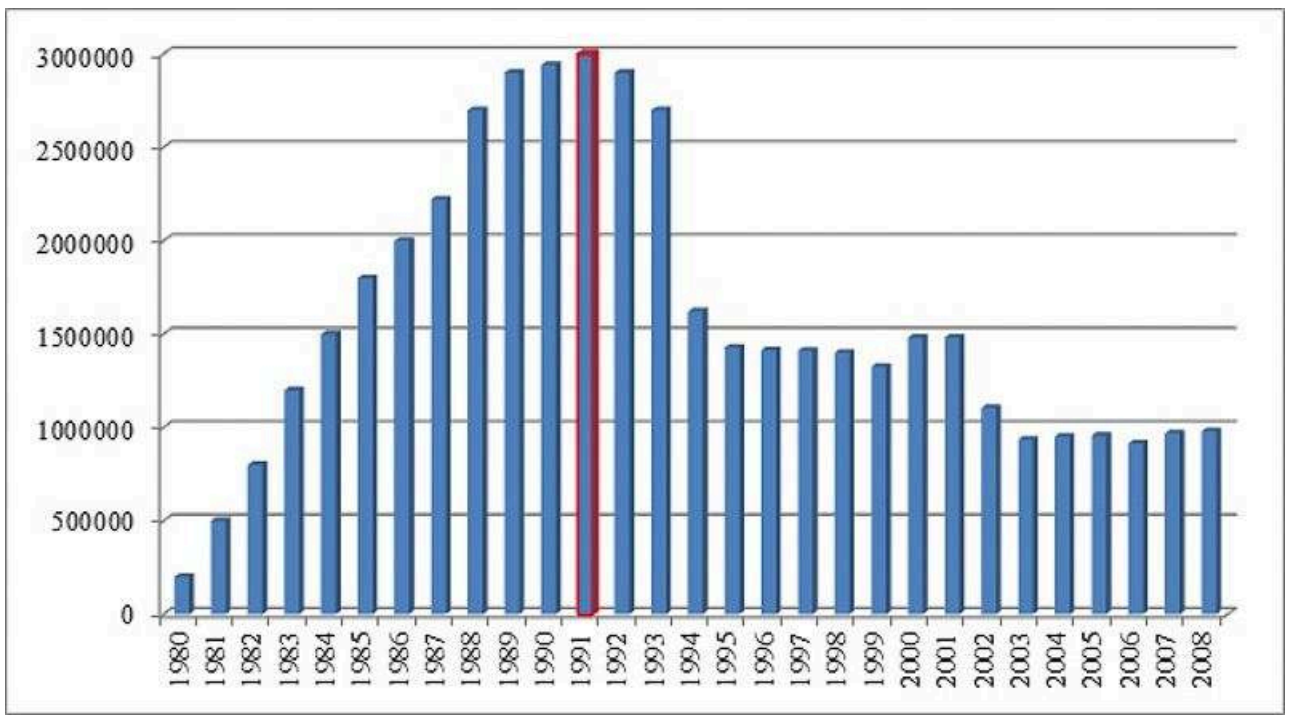

Source : UNHCR

15 Selon le HCR (2009-2016), quelques 900000 réfugiés afghans sont enregistrés (personnes relevant de la compétence du HCR) comme étant toujours en Iran. Seuls 27000 d'entre eux (soit $3 \%$ ) résident dans les zones prévues pour l'installation des réfugiés, celles gérées par le Bureau des étrangers et de l'immigration (BAFIA). Il faut y ajouter les clandestins (difficiles à dénombrer) ainsi que les travailleurs saisonniers qui passent quelques mois dans le pays avant de rentrer. Pour les immigrés afghans non 
enregistrés (sans papiers), l'Iran a mis en œuvre, à partir de mai 2010, un plan global de régularisation basé sur l'octroi de permis de séjour, inclus dans les passeports (des visas allant de trois mois à un an). Selon le BAFIA, un peu plus de 1,4 million d'Afghans ont participé et ont été enregistrés, dont 480000 ont obtenu le visa de travail valable pour un $a^{7}$, avec la « possibilité » du renouvellement mais « pas la garantie».

Pour mieux comprendre la diversité des faits migratoires afghans, Monsutti (2006) pointe le va-et-vient existant entre rapatriement et réitération de l'immigration; il insiste sur le fait que le retour en Afghanistan ne signifie pas nécessairement la fin des déplacements et qu'il peut initier d'autres mouvements migratoires, suivant un schéma de mouvements transfrontaliers multidirectionnels. Il existe en effet des réseaux transnationaux préétablis entre l'Afghanistan, le Pakistan et l'Iran, qu'il s'agisse de mouvements d'individus en quête du travail ou pour fuir la guerre.

Il ressort de la littérature existante sur les immigrés afghans en Iran au moins deux aspects essentiels : en premier lieu, elle met en avant la notion de réseau, procédant donc à l'étude de la migration afghane à travers l'ethnographie des réseaux sociaux. Parmi les auteurs développant cette notion, on peut notamment citer Alessandro Monsutti (2004, 2006 et 2007, 2009) et Harpviken (2009). Ces auteurs, et en particulier Monsutti, insistent à travers leurs enquêtes sur la nécessité d'aller au-delà d'une perception courante de la migration afghane comme causée par la guerre, en mettant en avant le fait que la migration n'est pas un processus unilinéaire. Grâce à une ethnographie itinérante, Monsutti a mis en œuvre une perspective transnationale qui éclaire ces pratiques et ces liens multiples. Sa description des territoires circulatoires des Afghans Hazara ${ }^{8}$ permet ainsi de relativiser la distinction entre migrations forcées et volontaires.

18 En deuxième lieu, diverses recherches ont principalement porté leur attention sur la vie des Afghans en Iran et sur la question du rapatriement et de ses obstacles. Le thème du rapatriement est en effet au cœur des débats et des recherches effectuées en Iran. En revanche, d'autres concepts comme l'intégration, l'identité ou l'adaptation sont peu mobilisés. Ils apparaissent dans certains titres mais sont rarement traités de façon approfondie. Concernant cette seconde composante, nous pouvons citer par exemple les travaux d'Abbasi-Shavazi et de ses collègues (2005a, b et c, 2006, 2008), ou ceux de Jamshidiha et Anbari (2004).

19 Par ailleurs, la documentation sur l'insertion des travailleurs immigrés ainsi que leur présence dans les espaces ruraux est quasiment absente; cela révèle que l'attention a essentiellement été portée sur les conséquences économiques de l'immigration pour le marché du travail national plutôt que sur l'insertion économique ou sociodémographique des immigrés (Amiri, 2017).

\section{Étude de cas}

20 Notre étude s'est essentiellement focalisée sur la période allant de 1978 à 2016, période marquée par des flux migratoires tant internes qu'externes. Et nous avons notamment étudié la région de Qazvin, elle aussi déficitaire en termes de solde migratoire parmi les zones rurales.

D'un point de vue méthodologique, cette étude se fonde, dans un première temps, sur un travail de terrain qualitatif par entretiens semi-directifs, menés à la fois avec des 
migrants internes et des immigrés afghans. Les entretiens ont été effectués entre mars et octobre $2010^{9}$. Un certain nombre de témoignages et d'observations complémentaires ont été récoltés en 2015 dans les lieux de départ et d'arrivée, en particulier à Karaj et à Téhéran. En d'autres termes, l'enquête suit un terrain multisitué. Dans un second temps, l'analyse s'appuie sur les données quantitatives des recensements iraniens afin de mieux comprendre les caractéristiques démographiques des sites étudiés et de mesurer les taux de migration.

Dans cette section, après avoir présenté le terrain et la problématique, nous tenterons, à partir de l'étude de cas, de creuser la question de la migration rurale-urbaine ainsi que de la migration afghane vers le milieu rural en utilisant les données du terrain.

\section{Choix des villages étudiés}

Après avoir examiné la carte des régions et des zones touchés par la migration, nous avons sélectionné certaines zones rurales dans la région de Qazvin, dans le Nord-Ouest de l'Iran: le village de Khiaraj dans la commune de Ràmand (département de Bouinzahra), le village de Khouznin dans la même commune, et Zinâbad dans la commune de Shâl. Le choix de ces zones rurales se justifie par le fait que ces villages étaient à la fois touchés par la migration interne et par la présence de travailleurs afghans. L'étude s'est focalisée sur le cas de Khiaraj qui était le plus touché (voir tableau 1 et illustration 9). Lors de l'enquête de terrain, Khiaraj comptait 69 habitants afghans, dont 36 hommes et 33 femmes, sur une population totale de 1012 habitants.

Une interview du gouverneur de la région de Qazvin éclaire la problématique dans cette région:

Dans les années précédentes, l'effectif des villages (Roustâ) de la région s'élevait à 1150 villages, mais le nombre des villages recule sensiblement et, aujourd'hui, nous sommes à 842 villages. Car 308 villages ont été complétement dépeuplés et donc nous devrions trouver une solution pour ce phénomène. ${ }^{10}$ (Agence de presse Mehrnews, le 19/2/2015).

Tableau 1 - Bref aperçu de la situation des sites sélectionnés

\begin{tabular}{|l|l|l|l|l|l|l|l|}
\hline \multirow{2}{*}{$\begin{array}{l}\text { Village } \\
\text { (Roustâ) }\end{array}$} & \multicolumn{2}{|l|}{ Population } & \multirow{2}{*}{$\begin{array}{l}\text { Canton rural } \\
\text { (Dehestân) }\end{array}$} & Commune & Département & Région \\
\cline { 2 - 8 } & Totale & Homme & Femme & & & \\
\hline Khiaraj & 1195 & 552 & 643 & Râmand Jonoubi & Râmand & Bouinzahra & Qazvin \\
\hline Khouznin & 2865 & 1417 & 1448 & Râmand Jonoubi & Râmand & Bouinzahra & Qazvin \\
\hline Zinâbad & 3958 & 1960 & 1998 & Zinâbad & Shâl & Bouinzahra & Qazvin \\
\hline
\end{tabular}




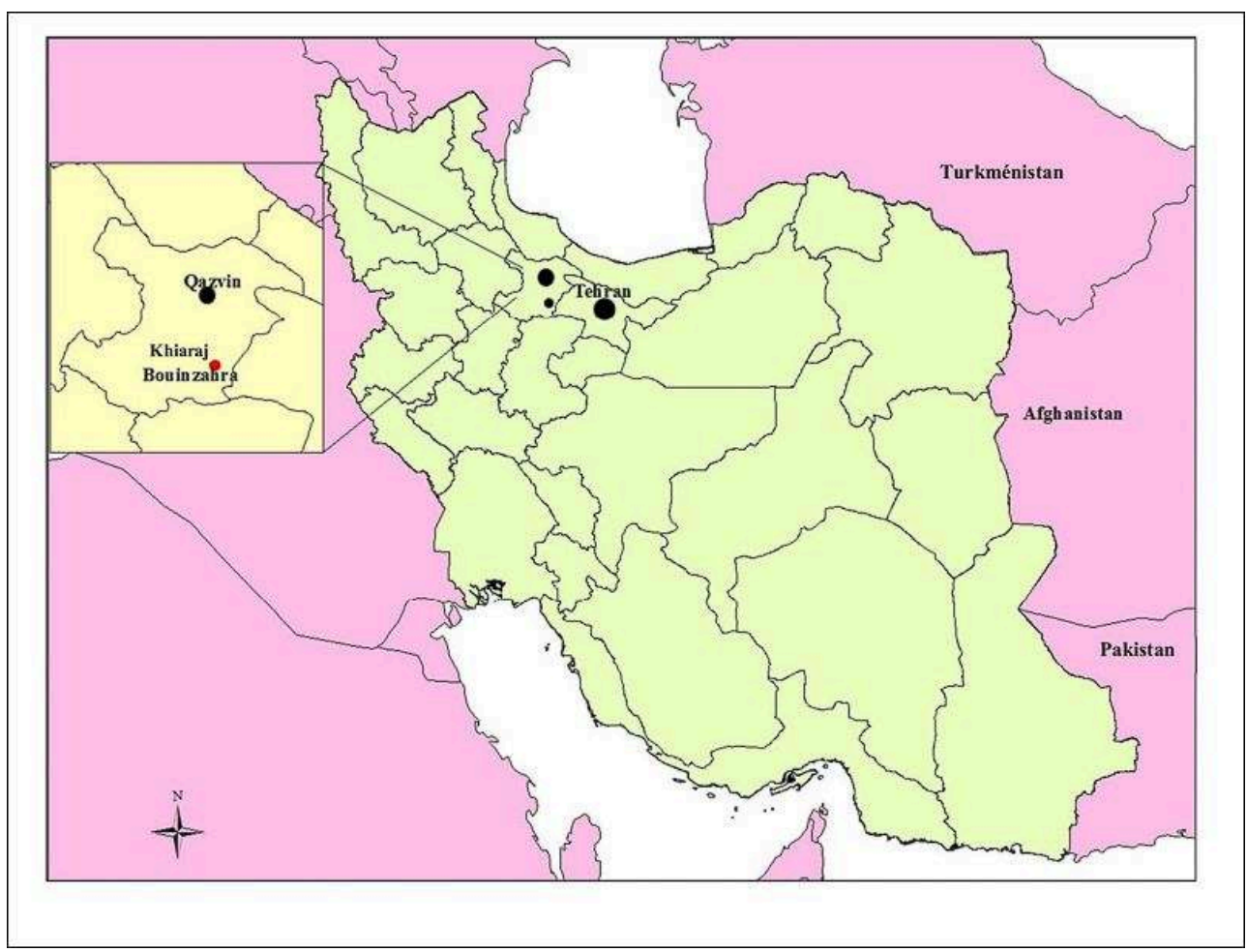

Auteur: Amir Amiri.

\section{Ampleur de la migration et structure démographique des zones étudiées}

Notre étude montre que la population des zones étudiées fait face à d'importants changements démographiques. Les migrants ruraux-urbains étant généralement des hommes, jeunes et mieux éduqués (sélectivité migratoire), la structure démographique de la population locale restante (celle des non-migrants) est composée majoritairement de femmes et de personnes âgées. À titre d'exemple, pour le village de Khiaraj, selon le recensement 2006, le rapport de masculinité était d'environ 92 hommes pour 100 femmes, et pour le village de Khoznin était de 97 hommes pour 100 femmes (illustrations 10 et 11).

En comparant les pyramides des âges des villages étudiés, l'effet des migrations à l'échelon local devient visible. En examinant la dernière pyramide des âges de Khiaraj en 2011, la première chose qui attire l'attention est un retrait ou une échancrure très significative au niveau des trois premiers groupes d'âge (0-14 ans) et des tranches d'âge moyen (35-49 ans). Ce retrait ne correspond pas à la mortalité, mais est lié aux départs d'adultes vers des zones urbaines. L'impact de la migration se sent aussi directement et indirectement sur la reproduction, parce que ces jeunes migrants adultes sont ceux en âge de procréer. En raison de leurs départs, la natalité a diminué. Ce phénomène de classes creuses entraîne à son tour l'étroitesse de la base de la pyramide des âges, qui est visible. 
Illustration 10 - Pyramide des âges de la population de Khiaraj

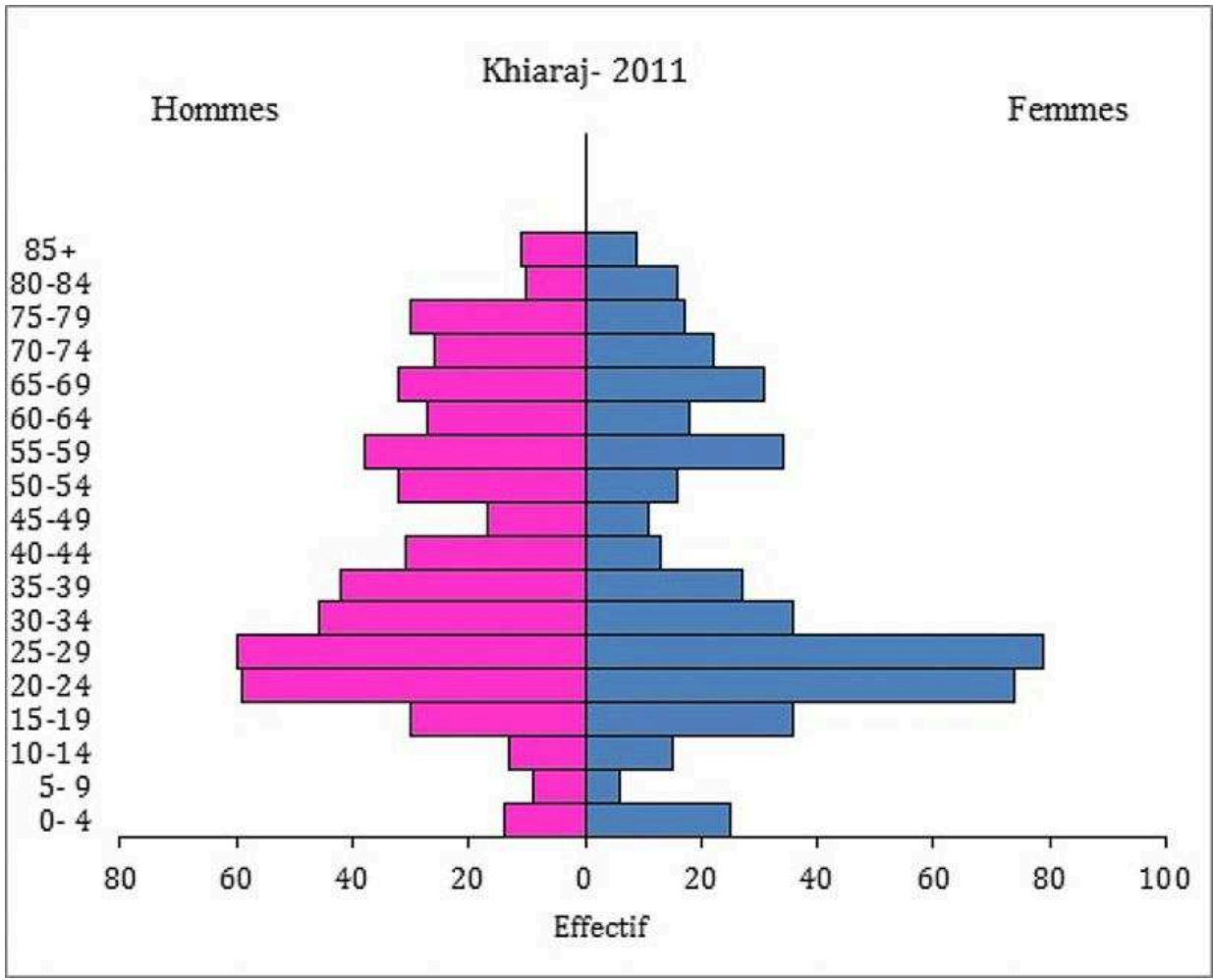

Source : Enquête de terrain, calculs de l'auteur à partir des données du centre de santé du village (markaz-é behdâsht).

Illustration 11 - Pyramides des âges de la population de Khoznin

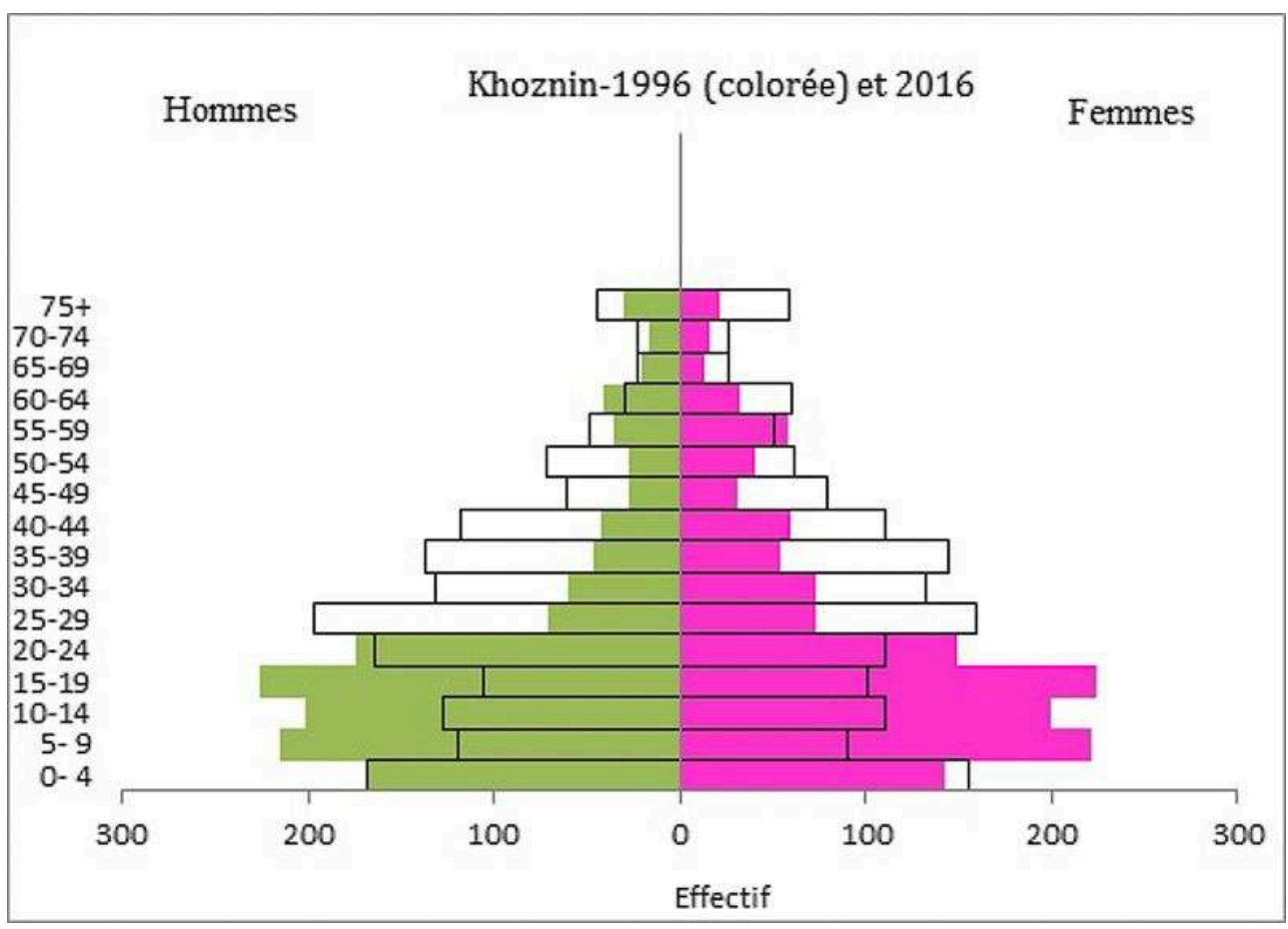

Source : Enquête de terrain, calculs de l'auteur à partir des données du centre de santé du village (markaz-é behdâsht). 
Illustration 12 - Pyramides des âges de la population urbaine et rurale de la région de Qazvin

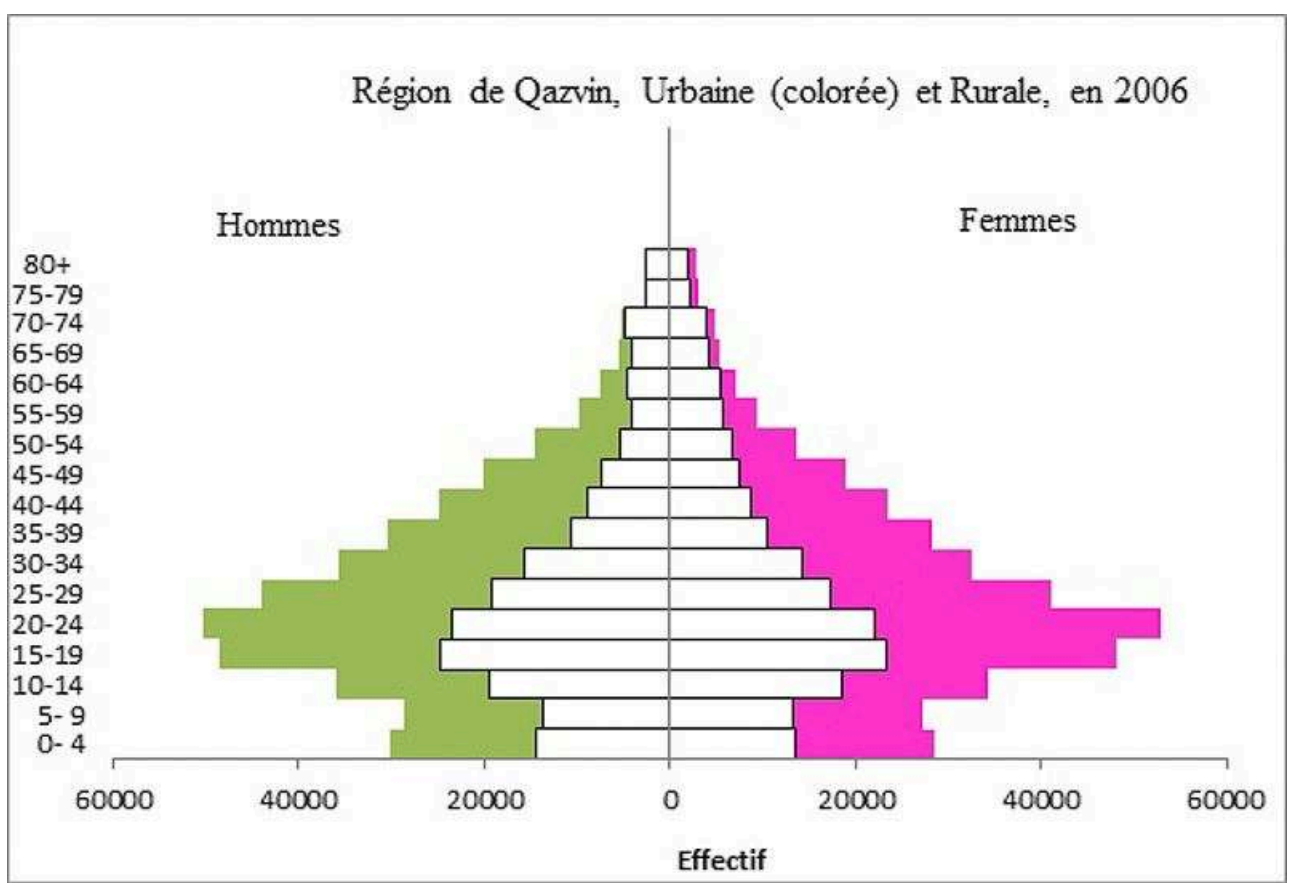

Source : Recensement de l'Iran, 2006.

Illustration 13 - Pyramides des âges de la population urbaine et rurale de l'ensemble de l'Iran, 2006

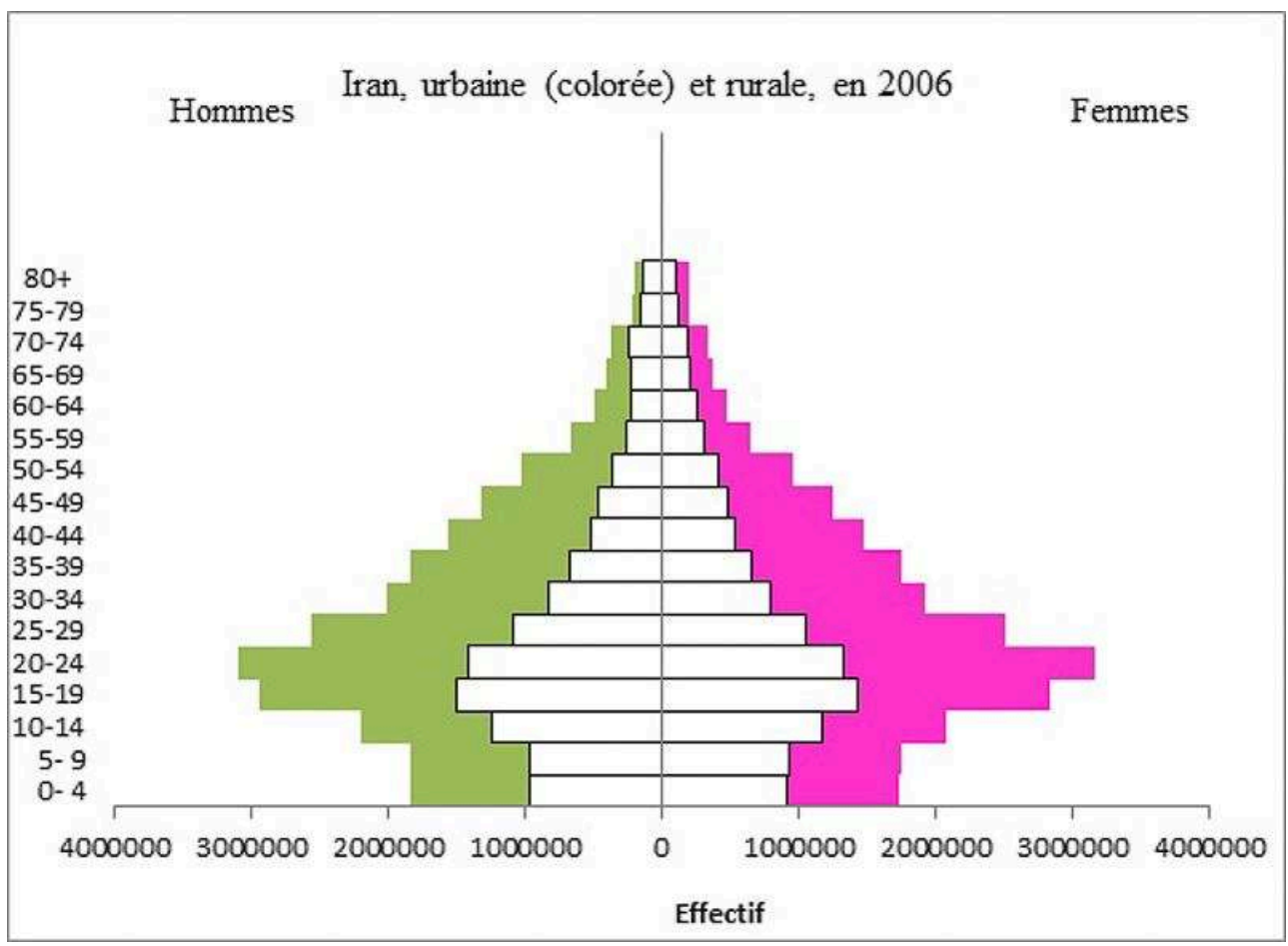

Source: Recensement de l'Iran, 2006. 


\section{Les causes principales de la migration interne}

Selon les résultats du recensement de 2011 (tableau 2), « les raisons familiales » comme l'accompagnement ou le regroupement familial (la " migration liée ») constituent $48 \%$ des causes de migrations internes en Iran. Ensuite, les raisons les plus courantes comprennent le travail, l'éducation et le service militaire. Cependant, la part des facteurs principaux varie selon le sexe. Les femmes migrantes sont plus concernées que les hommes par les facteurs " éducation » et " migration liée », tandis que les migrants masculins sont plus concernés par les facteurs tels que «travail» et «service militaire ».

Tableau 2 - Répartition relative des migrants internes selon la cause de la migration, le lieu de destination (direction) et le sexe, en 2011

\begin{tabular}{|c|c|c|c|c|}
\hline & \multicolumn{2}{|c|}{ Sexe (\%) } & \multicolumn{2}{|c|}{ Destination (\%) } \\
\hline & Homme & Femme & Urbaine & Rurale \\
\hline Recherche de travail & 11 & 2 & 7 & 7 \\
\hline Quête d'un meilleur travail & 7 & 1 & 4 & 4 \\
\hline Mobilité due au travail & 8 & 1 & 5 & 2 \\
\hline Éducation & 14 & 14 & 15 & 9 \\
\hline Service militaire & 11 & 0 & 4 & 12 \\
\hline Qualité du logement & 15 & 5 & 10 & 12 \\
\hline Accompagnement & 26 & 69 & 46 & 47 \\
\hline Autre & 6 & 6 & 7 & 5 \\
\hline Non déclaré & 2 & 2 & 2 & 2 \\
\hline Total & 100 & 100 & 100 & 100 \\
\hline Effectif & 2908560 & 2626106 & 4302086 & 1232580 \\
\hline
\end{tabular}

Source : Recensement de l'Iran, 2011.

Pour mieux comprendre la dynamique migratoire de départ et d'arrivée, il nous semble utile d'aborder la question du départ de la population locale vers les grandes villes. Nous appuyant sur les témoignages de terrain, nous avons exploré les facteurs qui y sont associés.

Pour la majorité des travailleurs locaux interrogés, la perspective de travailler sur des terres agricoles n'était plus une option attractive. L'agriculture ne représente plus ni la promesse d'un gain financier ni celle d'une indépendance personnelle suffisante par rapport aux opportunités d'emploi à l'extérieur de l'environnement habituel. 
30 Ainsi, Q, un homme iranien âgé de 41 ans, explique le motif de son départ pour Téhéran :

«Là-bas [dans le village], nous n'avons pas eu grand-chose à cultiver. Nous avons eu deux à trois hectares de terrain. Cette quantité de terres cultivées a été suffisante pour une petite famille et un seul paysan y suffisait, alors que nous étions une famille de sept enfants, cinq garçons et deux filles. Depuis mon plus jeune âge j'ai aidé mon père, je n'avais pas de revenu indépendant de mon travail agricole et mon travail était une partie des revenus de mon père et du revenu familial ». (Q, homme, 41 ans, travailleur iranien).

31 Cet interviewé, en expliquant que la raison principale de son départ était l'insuffisance de terres cultivables, couple celle-ci un autre facteur important: la volonté de s'émanciper. En effet, au sein de «l'agriculture familiale», l'organisation socioéconomique se base sur la mobilisation du travail familial sur les champs et sous l'autorité d'un individu, le « chef (père) » de famille. Ce travail, bien que transmissible de génération en génération, ne résiste pas au processus de modernisation. D'un côté, l'exploitation familiale ne supporte qu'une activité de subsistance ou de petite taille et peu productive. De l'autre, la terre étant généralement héritée à parts égales au sein des fratries, il suffit de quelques générations pour qu'une étendue de terre autrefois suffisante pour subvenir aux besoins d'une famille, devienne si morcelée qu'elle ne réponde plus aux besoins de ses membres. La migration apparaît dès lors comme un moyen de résoudre ce problème, les partants laissant souvent leurs terres à la disposition des membres de la famille qui restent au village.

32 En outre, pour la plupart des jeunes, le fait de limiter ses possibilités d'embauche au milieu rural équivaut à demeurer enfermé dans un cercle d'emplois limités, un choix dont l'horizon ne va pas au-delà des secteurs de l'agriculture et de l'élevage, les emplois ruraux non agricoles étant insuffisants.

«Après avoir fini mon service militaire, je ne résistais que 10 jours au village. Je ne voyais pas un avenir rassurant. Je voulais faire une formation de pâtisserie. [...] puis, le travail au village n'assurait pas de revenu suffisant, donc, je cherchais le bien-être, je rêvais d'avoir une maison, d'épargner de l'argent, d'avoir des investissements, d'avoir une voiture, avoir une pension et des assurances, pour avoir assez d'économies pour cinquante ans et au-delà ». (AH, homme âgé de 41 ans, migrant rural habitant à Karaj ${ }^{11}$ depuis 20 ans).

Ce témoignage montre que la ville nourrit la perspective d'emplois mieux rémunérés avec une possibilité d'ascension sociale répondant donc mieux aux aspirations socioéconomiques et culturelles. Ici, on peut également se référer au rôle des facteurs répulsifs des villages et attractifs des villes (push-pull factors).

Dans ce milieu, un autre facteur influent était la taille du ménage. Les ménages ayant une main-d'œuvre excédentaire et disponible étaient obligés de trouver des emplois au-delà de la communauté immédiate. De même, les ménages qui ont besoin de revenus supplémentaires doivent chercher ailleurs pour répondre à leurs besoins. Ceci est bien montré dans le témoignage de $M$ et $M O$ :

"J'ai grandi dans une famille de huit enfants et nous avons eu environ trois hectares de terrain. Nous étions trois frères, et maintenant nous travaillons tous les trois à la ville. Notre terrain est cultivé par mon père ». (M, spécialiste de plâtrage, âgé de 50 ans, migrant à Téhéran).

De même, MO souligne lui aussi la gravité de la question : 
« Nous étions une famille de neuf membres, nous avions dix hectares de terrain et environ un hectare de jardin et un vignoble aussi. Dans notre famille, mes quatre frères aînés devant moi ont quitté le village pour le travail et leurs études». (MO, homme âgé de 32 ans, migrant rural à

Téhéran). classiques de la migration rurale-urbaine expliquent également une partie de la question. Les facteurs tels que le mode de vie, le capital social, le mariage, l'éducation ainsi que les infrastructures culturelles et les services sociaux des villes, sont reconnus comme les facteurs incitant au départ.

\section{L'immigration Afghane en milieu rural}

En plus des demandeurs d'asile et des réfugiés à la suite des conflits périodiques en Afghanistan, il est important de souligner que, depuis plus de quatre décennies, l'agriculture a été un point d'entrée dans le marché du travail pour les immigrants en Iran (tableau 3). À titre d'exemple, les données du recensement iranien de 2006 illustrant la répartition des actifs afghans âgés de 10 ans et plus selon les principaux groupes professionnels nous montrent que plus de $16 \%$ des actifs immigrés (Afghans) travaillent dans le secteur agricole. Pour les immigrants, l'emploi dans le secteur agricole occupe la troisième place, juste derrière les secteurs du bâtiment (40\%) et de l'industrie (20\%).

Tableau 3 - Répartition des actifs afghans âgés de 10 ans et plus selon les principaux groupes professionnels, niveau national, 2006

\begin{tabular}{|l|l|l|l|l|}
\hline \multirow{2}{*}{} & \multicolumn{3}{|l|}{ actifs afghans } & \multicolumn{2}{l|}{$\begin{array}{l}\text { actifs } \\
\text { autochtones }\end{array}$} \\
\cline { 2 - 6 } & Effectifs & $\%$ & Effectifs & $\%$ \\
\hline agriculture, chasse et sylviculture & 66024 & 16,15 & 3457114 & 17,82 \\
\hline pêche & 203 & 0,05 & 72842 & 0,38 \\
\hline exploitation minière & 404 & 0,10 & 141255 & 0,73 \\
\hline
\end{tabular}

EchoGéo, 45 | 2018 


\begin{tabular}{|l|l|l|l|l|}
\hline industrie & 82210 & 20,11 & 3341509 & 17,22 \\
\hline approvisionnement en électricité, gaz et eau & 1029 & 0,25 & 221568 & 1,14 \\
\hline bâtiment/construction & 165463 & 40,48 & 2418488 & 12,47 \\
\hline $\begin{array}{l}\text { grossiste, détaillant, opérateurs et monteurs de moyens de } \\
\text { transport et d'articles personnels }\end{array}$ & 49918 & 12,21 & 2747958 & 14,17 \\
\hline hôtellerie et restauration & 808 & 0,20 & 186287 & 0,96 \\
\hline transport, stockage et communications & 8209 & 2,01 & 1923552 & 9,92 \\
\hline entremise financière & 219 & 0,05 & 277807 & 1,43 \\
\hline locations et commerce & 3446 & 0,84 & 420886 & 2,17 \\
\hline affaires publiques, défense et sécurité sociale obligatoire & 8507 & 2,08 & 1434696 & 7,40 \\
\hline instruction / éducation & 2805 & 0,69 & 1323471 & 6,82 \\
\hline sanitaire et assistance sociale & 957 & 0,23 & 511269 & 2,64 \\
\hline autres activités de services publics, sociaux et personnels & 17226 & 4,21 & 883366 & 4,55 \\
\hline Total & 408788 & 100 & 19396417 & 100 \\
\hline
\end{tabular}

Source : Calculs de l'auteur à partir des données bruts du recensement général de la population et de I'habitat en 2006, Centre de statistiques de l'Iran.

\section{Métiers ouverts aux Afghans}

Pour accéder au marché du travail, plusieurs défis se posent aux étrangers et en l'occurrence, aux Afghans. Le travail ne leur est pas interdit par la loi, mais il est conditionné à l'observance de règles bureaucratiques compliquées. L'accès au marché du travail formel s'avère donc en général ardu. Les emplois qualifiés leur sont interdits pour des raisons statutaires ou juridiques, ce qui peut expliquer non seulement leurs difficultés d'insertion sur le marché du travail, mais aussi leur concentration dans certains secteurs d'activité " ouverts/autorisés ». Cette liste comprend des métiers qui sont principalement concentrés dans le secteur du bâtiment et des travaux publics, de l'agriculture, etc. Dans un tel contexte limitatif (difficultés d'obtenir le permis de travail ou le renouvellement du titre de séjour), il est envisageable que, une fois installés, les immigrants s'orientent vers l'économie informelle et dans les secteurs où la pénurie de main d'œuvre est reconnue. Il importe également de noter que, en raison de la nature des métiers ruraux qui implique un séjour hors des zones urbaines plus contrôlées, de nombreux immigrants, en particulier ceux qui séjournent 
clandestinement ou les saisonniers, sont attirés par ces emplois, dès lors moins risqués ${ }^{12}$.

Pour mieux comprendre la variété de leurs travaux, nous avons essayé de regrouper les activités des immigrants afghans vivant dans les villages étudiés en catégories : travaux agricoles (irrigation, conducteur de tracteur, récolte de blé, récolte de tournesol et autres produits agricoles, etc.); travail dans la construction (ouvrier/maçonnerie, etc.) ; activités liées à l'élevage et à la volaille (bergerie, partenariat en élevage, etc.) ; enfin autres travaux dispersés (comme le chargement/déchargement des camions, la collecte des ordures, le gardiennage, etc.).

\section{Sur le terrain et le marché de l'emploi}

La plupart des immigrés afghans en activité s'inscrivent en fait dans les catégories des agriculteurs exploitants, des éleveurs de bétail ou de volaille et dans des métiers similaires ou bien dans des professions connexes. Les autres trouvent à se faire embaucher dans le secteur des travaux de construction. Parmi les immigrés habitant à Khiaraj, le travail le plus fréquent était celui d'ouvrier dans le secteur agricole comme l'irrigation des champs, la récolte etc., aussi bien que les travaux d'élevage.

Concernant les différents travaux qu'ils ont accompli, les enquêtés afghans en milieu rural nous en parlent :

Un jour, monsieur Z., habitant de ce village, est venu à la ville de Takestan, à la recherche d'ouvriers où nous attendions du travail. [...], après nous avons été familiarisés avec l'environnement d'ici, nous avons senti que le peuple de Khiaraj est accueillant, il y a du travail et ils nous ont demandé de venir et de rester aussi. Donc, nous nous sommes installés. [...], Au début, j'étais un ouvrier simple, mais maintenant cela fait trois ans que je suis éleveur ovin. (V., 52 ans, homme afghan travailleur à Khiaraj).

Ce cas témoigne d'un besoin du marché du travail rural qui, compte tenu d'un environnement favorable, l'ont poussé à rester. Mais, Z., un autre enquêté afghan en milieu rural, met en avant le rôle de son réseau dans son installation au village :

Avant de venir à Khiaraj, les quatre premières années, j'étais à la ville de Robatkarim, où je travaillais dans un poulailler d'élevage en batterie, puis pendant cinq à six ans, j'ai vécu à Takistan. Maintenant, il y a dix ans que je suis à Khiaraj. Nous sommes venus ici grâce à monsieur V. En fait, il était le premier Afghan venu à Khiaraj. Maintenant, je suis berger. (Z., homme afghan âgé de 50 ans, travailleur à Khiaraj).

Ainsi, même si les itinéraires empruntés divergent, l'immigration depuis un même lieu d'origine ou parmi les membres d'un même groupe ethnique vers un même lieu d'arrivée/d'accueil s'accompagne et passe par le tissage de réseaux de relations. Ceci est une règle assez courante dans les dynamiques migratoires. En effet, à leur arrivée, ils passent souvent chez les proches, qui les aident à trouver un travail et un logement. Il n'est donc pas surprenant que les migrants obtiennent des emplois dans les mêmes métiers que leurs proches et, au fur et à mesure, forment avec les autres immigrés de même origine, des groupes qui s'installent dans les mêmes villages ou dans les mêmes quartiers, ou, s'ils s'installent dans les zones urbaines, généralement en banlieue ou à la périphérie des grandes villes. 
Cependant, les mouvements migratoires sont loin d'être tous linéaires. L'histoire de monsieur DA en témoigne :

Je me suis marié il y a 22 ans, avant ce moment-là, j'ai vécu cinq ans en Iran. La première fois (il y a 27 ans) j'ai traversé la frontière à pied et je suis arrivé clandestinement à Mashhad, puis j'ai déménagé à Téhéran, pendant environ trois ans dans les montagnes de Téhéran (Damavand), j'ai été berger. Par la suite, pendant environ un à deux ans, j'ai travaillé à Téhéran sur les chantiers de construction et puis je suis allé en Afghanistan. Je suis resté six mois en Afghanistan. Plus tard, avec ma famille nous avons reimmigré en Iran et je travaille sur des vignobles. (DA, 55 ans, homme afghan travaillant à Khiaraj).

Ces témoignages mettent en évidence l'aspect saisonnier et circulaire du travail en milieu rural des afghans. Ce modèle de mobilité manifeste le caractère continuel et cyclique de la migration, caractère qui a brouillé les lignes entre "réfugiés » et immigrants « volontaires ", selon le temps et le lieu.

Pour être synthétique, au vu de nos observations, les formes courantes de travail de la zone étudiée et ses caractères différentiels sont les suivants :

- L'emploi occasionnel et saisonnier comporte une mobilité élevée et une insertion transitoire. Le cas le plus typique est celui du travail d'ouvrier agricole régularisé ou non, employé au moment des récoltes qui demandent une utilisation intensive de main d'œuvre sur une courte période. Ils travaillent au jour le jour. Il suppose également une forte mobilité spatiale, des va-et-vient avec le pays d'origine, des tentatives de combinaison avec d'autres emplois, eux aussi occasionnels.

- L'emploi semi-continuel comporte une certaine continuité de rapport avec le même employeur, mais cette relation est intermittente, permettant de couvrir les pics de la demande et les besoins périodiques. Une bonne partie de l'insertion dans le bâtiment ou la construction et les activités qui leur sont liées ainsi que l'élevage, associé à un investisseur local, relève de cette catégorie.

- Le travail indépendant et l'auto-emploi concernent des immigrés qui ont la possibilité d'investir dans des activités indépendantes, notamment dans l'élevage du bétail.

\section{Effet de vases communicants}

Enfin, nous nous sommes penchés sur la réaction des migrants locaux concernant la présence des Afghans. Pour ce faire, encore une fois, nous avons posé une question aux enquêtés locaux (migrants ruraux identifiés à l'aide des réseaux locaux et interviewés à Téhéran et Karaj) sur leur décision de partir et par conséquent de voir une force de travail étrangère les remplaçant dans leur village. La prise de position des migrants était plutôt favorable au travail des Afghans dans leur village. Ainsi, $Q$., conscient de la situation, s'exprime :

Étant donné que la main-d'œuvre locale est partie et que beaucoup de gens ont vieilli, ils doivent employer des Afghans. Je ne me sens pas bien quand je vois que le village est vide, mais s'ils (les Afghans) n'étaient pas là, il serait difficile de cultiver. (Q., homme âgé de 41 ans, migrant iranien à Téhéran).

De même, d'autres enquêtés, nous ont d'abord manifesté leur sentiment de regret que leur village soit devenu vide, mais en même temps, reconnaissaient être partis définitivement, sans espoir de retour, ne voyant dès lors pas d'autre solution pour leur 
village d'origine que de bénéficier de la force du travail afghane, n'étant pas prêts à vivre et travailler en vase clos.

\section{Conclusion}

51 Les données que nous avons recueillies dans notre étude de cas suggèrent comment à la fois la migration interne et la migration internationale se déroulent selon des chronologies différentes, mais connexes, mettant donc en évidence leur relation. Ainsi, une des principales conclusions est d'expliciter ce lien entre les deux types de migration. Tandis que les raisons principales de quitter les zones rurales sont le chômage ou la recherche de meilleurs emplois et le désir d'améliorer ses conditions de vie, de l'autre côté, un phénomène intéressant s'est produit faisant que le site étudié est devenu attractif pour les immigrants, non pas pour la prospérité relative de la région, mais pour occuper les postes restant vacants, et dans une certaine mesure, en leur permettant de contourner des barrières administratives de l'emploi en ville. En d'autres termes, le vide laissé par les travailleurs ruraux est rempli par les travailleurs étrangers qui sont essentiellement des immigrés afghans. Sur cette base, dans certains contexte (sans généraliser), la migration interne est une condition préalable des flux internationaux, sans tenir compte les causes de la migration involontaire. Enfin, compte tenu de ce qui précède, nous pensons que les processus de la mobilité ruraleurbaine à long terme peut inciter des flux migratoires en direction opposée vers les zones rurales.

\section{BIBLIOGRAPHIE}

Abbasi-Shavazi M. J., 2006. État des immigrés internationaux et des réfugiés dans la République islamique d'Iran. Téhéran, UNFPA et HCR, 88 p.

Abbasi-Shavazi M.J., Glazebrook D., Jamshidiha G., Mahmoudian H., Sadeghi R., 2005a. Return to Afghanistan? A Study of Afghans Living in Tehran. Kabul, Afghanistan Research and Evaluation Unit, 66 p. [En ligne]. http://www.unhcr.org/430b1c842.pdf

Abbasi-Shavazi M.J., Glazebrook D., Jamshidiha G., Mahmoudian H., Sadeghi R., 2005b. Return to Afghanistan? A Study of Afghans Living in Zahedan, Islamic Republic of Iran. Kabul, Afghanistan Research and Evaluation Unit, 76 p. [En ligne]. http://www.unhcr.org/4430da902.pdf

Abbasi-Shavazi M.J., Glazebrook D., Jamshidiha G., Mahmoudian H., Sadeghi R., 2005c. Return to Afghanistan? A Study of Afghans Living in Mashhad, Islamic Republic of Iran. Kabul, Afghanistan Research and Evaluation Unit, 80 p. [En ligne]. http://www.unhcr.org/434f69e92.pdf

Abbasi-Shavazi M.J., Glazebrook D., Jamshidiha G., Mahmoudian H., Sadeghi R., 2008. Secondgeneration Afghans in Iran: Integration, Identity and Return. Kabul, Afghanistan Research and Evaluation Unit, 92 p. [En ligne]. https://areu.org.af/wp-content/uploads/2016/01/823E-SecondGeneration-Afghans-in-Iran-CS-2008.pdf 
Amiri A. M., 2017. Entre inclusion et exclusion des immigrés : le cas des Afghans en Iran. Facteurs démographiques, sociaux et politiques. Thèse de doctorat en Démographie et Sciences Sociales, Paris, EHESS.

Bureau des affaires des étrangers et immigrés, 2003-2012. Le recensement et l'organisation spatiale des ressortissants Afghans (Tarh-é Âmâyesh-é atbâ-é Afghani).

Centre de Statistiques d'Iran, 1976-2016. Résultats détaillés des recensements de 1976, 1986, 1996, 2006 et 2011 et 2016 (Natâyej-é tafsili-yé Sarshomari-yé Omoumi-yé Nofous va Maskan).

Farazmand A., 2001 [2 ${ }^{\text {nd }}$ ed. updated]. Bureaucracy, Agrarian Reforms, and Regime Enhancement: The Case of Iran. In Farazmand A. (ed.), Handbook of Comparative and Development Public Administration. New York, Marcel Dekker Inc., Chapter 39, p. 551-565.

Harpviken K. B., 2009. Social Networks and Migration in Wartime Afghanistan. Houndmills, Palgrave, $226 \mathrm{p}$.

HCR, 1994-2010. Annuaires Statistiques.

IRIB [Agence de presse iranienne], 2013. Interview du directeur de BAFIA, 25/08/2013 [En ligne]. http://www.iribnews.ir/fa/news/35448/15

Jamshidiha G., Anbari M., 2004. Taàlloqat-é ejtemaee va asarât-é ân bar bâzgasht-é mohajerin-é Afghani, (Attachements sociaux et leurs effets sur le rapatriement des immigrés afghans en Iran) Nameh é oloum é ejtemaee, shomareh é 23, p. 43-68 [En persan].

Karimi Moughari, Z., 2007. The effects of afghan immigrants on the Iranian labour market. Iranian Economic Review, Vol.13, $\mathrm{n}^{\circ}$ 20, p. 57-84.

Lahsaeizadeh A., 1993. Contemporary rural Iran. Aldershot, Avebury, 360 p.

Monsutti A., 2004. Guerres et Migrations, Réseaux Sociaux et Stratégies Economiques des Hazaras d'Afghanistan. Paris/Neuchâtel, Editions de l'Institut d'Ethnologie, Editions de la Maison des Sciences de l'Homme, $364 \mathrm{p}$.

Monsutti A., 2006a. Afghan Transnational Networks: Looking Beyond Repatriation. Commisioned report. Kabul, Afghanistan Research and Evaluation Unit, 52 p. [En ligne]. https://reliefweb.int/sites/ reliefweb.int/files/resources/6A583751924F6A31492571F400099A52-areu-afg-31aug.pdf

Monsutti A., 2006b. Au-delà de la figure du réfugié : la reproduction des liens sociaux en situation migratoire (le cas afghan). In Mohammad-Arif A. et Schmitz J. (dir.), Figures d'Islam après le 11 septembre. Disciples et martyrs, réfugiés et migrants. Paris, Karthala, p. 227-256.

Monsutti A., 2007. Migration as a rite of passage: Young Afghans building masculinity and adulthood in Iran. Iranian Studies, vol. 40, n 2, p. 167-185.

Monsutti A., 2009. Itinérances transnationales : un éclairage sur les réseaux migratoires afghans. Critique internationale, 44, p. 83-104.

Mohmoudian H., 2016. Internal migration and urbanization in Iran: status, challenges and policy guidelines. European Population Conference (EPC), Mainz, 31st August to 3rd September 2016.

Mohmoudian H., Ghassemi-Ardahaee A., 2014. Internal migration and urbanization in I.R. Iran. Tehran, University of Tehran and the United Nations Population Fund.

UNHCR, 1997. Afghanistan : une crise qui s'éternise. Réfugiés [En ligne], n¹08. http:// www.unhcr.org/fr/publications/refugeemag/4ad2f89910/magazine-refugies-n\%C2\%B0-108afghanistan-crise-seternise.html 
UNHCR, 2001. Afghan Refugee Statistics. [En ligne]. https://reliefweb.int/report/afghanistan/ unhcr-afghan-refugee-statistics-10-sep-2001

UNHCR, 2009. Appel global 2009 du HCR, République islamique d'Iran [En ligne] http:// www.unhcr.org/fr/4ad2f717f.pdf

UNHCR, 2011, UNHCR Global Trends 2010. [En ligne]. http://www.unhcr.org/statistics/country/ 4dfa11499/unhcr-global-trends-2010.html

UNHCR, 2013. Le nombre de déracinés le plus élevé de ces 18 dernières années. [En ligne]. http:// www.unhcr.org/fr/news/stories/2013/6/51c08160c/nombre-deracines-eleve-18-dernieresannees.html

UNHCR, 2016. Afghan solutions strategy. [En ligne]. http://www.unhcr.org/afghan-solutionsstrategy.html

\section{NOTES}

1. La Révolution Blanche (en persan : Enghelab-e-Sefid) est une série de réformes à grande portée lancée en 1963 sous la dynastie Pahlavi II, destinée à moderniser l'économie et la société iranienne, au cœur de laquelle était la réforme agraire. Dans ce contexte, l'inégale redistribution des terres et la fragmentation des propriétés sont des facteurs qui ont conduit à la paupérisation de la société rurale. Les travailleurs agricoles qui ne possédaient pas de droit « de cultiver des terres » (Khoshnéshin) ont été les grands perdants de la réforme agraire et souvent ont été amenés à quitter les villages pour les villes (Lahsaeizadeh, 1993).

2. Cette politique étant renforcée par les opérations efficaces d'organismes de la «Croisade pour la reconstruction " (Jahad-e-Sazandegi), chargés du développement et de l'équipement de ces zones rurales ainsi que d'autres organisations telles que la Fondation du logement (Bonyand-eMaskan), qui fournissait des logements pour les ménages à faibles revenus en accordant des prêts (par l'intermédiaire des banques) et les commissions de sept membres pour la concession et la mise en culture des terres (Heiathay-e-haft nafare vâgozâri-e va ehya-é arazi).

3. Selon la définition de l'INSEE (2016), le solde migratoire est la différence entre le nombre de personnes qui sont entrées sur le territoire et le nombre de personnes qui en sont sorties au cours de l'année. Ce concept est indépendant de la nationalité.

4. Devant les déplacements des Irakiens, qui comptent aussi parmi les autres flux importants.

5. Dans cet article, nous utiliserons le terme "immigrés » dans son sens général qui englobe les différentes catégories d'immigrés y compris les « réfugiés ".

6. En 2001, après la chute du régime des talibans, les Afghans restaient sceptiques et n'avaient guère la volonté de retourner dans leur pays. La même année, les salaires moyens perçus par les travailleurs occasionnels dans les grandes villes d'Afghanistan avaient diminué de 4 à $49 \%$ par rapport à l'année précédente. Le salaire mensuel à Hirat était de $15 \$$ par mois, alors que le salaire moyen des travailleurs afghans en Iran était supérieur à 100 \$ (Karimi Moughari, 2007). Cela illustre qu'en effet, les motifs de départ peuvent être toujours multiples et complexes et bien souvent liés entre eux. S'y mêlent la violence politique ou religieuse, la faillite politique et économique ou l'absence de perspective d'avenir.

7. Interview du directeur de BAFIA par l'Agence de presse iranienne IRIB, le 25/08/2013.

8. Les Hazara sont l'un des principaux groupes ethniques de l'Afghanistan et ont pour principale marqueur identitaire le fait d'être musulmans chiites dans un pays majoritairement sunnite.

9. Cet article est extrait d'un chapitre d'une thèse de doctorat portant sur l'inclusion et l'exclusion des Afghans en Iran (Amiri, 2017). 
10. Il est important de définir ce que l'on appelle Roustâ et Dehestân. Le premier est un village, regroupant au minimum 20 ménages soit une centaine d'habitants. Le Dehestân, lui, est une unité administrative composée de plusieurs villages, constituant la plus petite division administrative rurale de l'Iran. Il est au-dessus du village et sous le Bakhsh (Commune/Division). La densité de population y oscille entre 4000 et 8000 habitants.

11. Le chef-lieu de la région Alborz.

12. Le paragraphe $\mathrm{c}$ de l'article 11 de la loi sur la réglementation financière de l'État, prévoit qu'afin d'éviter la présence non autorisée de travailleurs étrangers sur le marché du travail, le ministère du travail et des affaires sociales doit punir les employeurs qui embauchent des travailleurs étrangers sans permis. Ainsi, pour chaque jour d'emploi illégal de travailleurs étrangers, une contravention équivalente à cinq fois le salaire minimum journalier est infligée et, en cas d'infractions répétées, l'amende sera doublée. Les dispositions du présent article ne comprennent pas l'emploi des ressortissants étrangers dans leurs camps de détention ou résidant dans les camps de réfugiés.

\section{RÉSUMÉS}

À travers une étude de cas, cet article propose de mieux comprendre les faits migratoires en Iran, tant du point de vue de la migration rurale-urbaine (en prenant pour exemple le Nord-Ouest) que de la relation de celle-ci avec l'immigration internationale (en traitant de la migration des Afghans dans le pays). Au cours de notre enquête de terrain sur les sites ruraux, les données empiriques recueillies suggèrent que la migration interne et la migration internationale se déroulent en effet de façon connexe. Une fois la réserve de migrants internes épuisée, le marché de l'emploi se tourne alors vers une source externe, de sorte que la migration interne peut apparaître comme une condition préalable aux flux internationaux. Et si la première relève des modèles classiques de la migration, l'hypothèse est que la seconde est alimentée de flux mixtes et circulaires.

Through a case study, this article proposes to better understand migration facts in Iran, both from the perspective of rural-urban migration (using an area in the Northwest as an example) and its relationship with international immigration (addressing Afghan migration in the country). During our field survey of rural sites, empirical data collected suggest that internal and international migrations do indeed take place in a related way. Once the supply of internal migrants has been exhausted, the labour market then turns to an external source, so that internal migration may appear to be a prerequisite for international flows. And while the former is based on traditional migration models, the hypothesis is that the latter is fed by mixed and circular flows. 
INDEX

Mots-clés : Dynamique migratoire, espace rural, migration interne, migration internationale, Iran, Afghan

Keywords : Migration dynamic, rural area, internal migration, international migration, Iran, Afghan

Thèmes : Sur le Champ - Sur le Terrain

\section{AUTEUR}

\section{AMIR AMIRI}

Amir Amiri, amir.amiri@ined.fr, est post-doctorant à l'Ined, rattaché à l'unité Parcours et territoires. 